\title{
Systematic Pan-Cancer Analysis Reveals the Prognostic Value and Immunological Role of EPHX2
}

\author{
Weiquan Hu \\ Ganzhou People's Hospital \\ Qinfei Zhao \\ First Affiliated Hospital of Gannan Medical University \\ Jing Xu \\ Sun Yat-Sen Memorial Hospital, Sun Yat-Sen University \\ Huaying Li \\ Xiangtan Medicine \& Health Vocational College \\ Longyu Zhu \\ The Fourth Hospital of Hebei Medical University \\ Suping Hu \\ First Affiliated Hospital of Gannan Medical University \\ Juan Li \\ Gannan Healthcare Vocational College \\ Fangfang Chen ( $\nabla$ longysh@163.com ) \\ Institute of Clinical Laboratory, Jinling hospital
}

\section{Research Article}

Keywords: EPHX2, pan-cancer, prognosis, immune infiltration, ICB

Posted Date: January 3rd, 2022

DOI: https://doi.org/10.21203/rs.3.rs-1081949/v2

License: (c) (i) This work is licensed under a Creative Commons Attribution 4.0 International License. Read Full License 


\section{Abstract \\ Background}

Accumulating evidence indicates the essential role of EPHX2 in tumorigenesis. However, to date, no studies have performed a systematic evaluation of EPHX2 gene in human cancers and the predictive role of EPHX2 in cancer immunotherapy response has still not been explored.

\section{Methods}

In the present study, Oncomine, TIMER2, UALCAN, GEPIA2, PrognoScan, HPA and Kaplan-Meier Plotter database were utilized to comprehensively analyze the expression landscape and prognostic clinical value of EPHX2 across 33 human cancers. To gain a better understanding of the role of EPHX2 in cancer immunotherapy, the correlations between EPHX2 and tumor immune microenvironment (TME) such as immune cell infiltrations, immune modulators, and the major histocompatibility complex were demonstrated. The underlying EPHX2-associated signaling pathways in cancer were also analysed. Moreover, the correlation between EPHX2 and immunotherapeutic biomarkers such as tumor mutational burden (TMB) and microsatellite instability (MSI) was explored. At last, the potential immune checkpoint blockers (ICB) response was predicted using tumor immune dysfunction and exclusion (TIDE) algorithm.

\section{Results}

Overall, the mRNA expression of EPHX2 was significantly downregulated in the majority of tumors compared with normal tissues. Despite the significant prognostic value of EPHX2 expression across cancers, EPHX2 played a protective or detrimental role in different kinds of cancers. Generally speaking, immune cell infiltrations, immune modulators and immunotherapeutic biomarkers were all strongly related to the expression of EPHX2. Besides, EPHX2 expression was significantly related to immunerelevant pathways, especially in PAAD, THYM and UVM. Furthermore, our study demonstrated diverse response patterns of ICB in response to EPHX2 expression in different tumor types.

\section{Conclusion}

Our findings here suggest that EPHX2 could be a prognostic factor in multiple cancers and play an important role in tumor immunity by affecting infiltrating immune cells, TMB and MSI. This study provides further insight into the role of EPHX2 in tumor immunotherapy.

\section{Background}

In the past few decades, cancer is rapidly becoming the number one killer in the world and a major barrier to significantly affect quality of life. To date, no effective treatment for cancer is expected to be available[1]. Nowadays, cancer immunotherapy especially ICB therapy has emerged as a promising cancer treatment[2]. With the continuous development and improvement of public databases such as The Cancer Genome Atlas (TCGA), bioinformatics has been extensively applied in many research fields. New immunotherapeutic targets for cancer can be found through pan-cancer expression analysis of genes and the assessment of their relevance to clinical prognosis and related signaling pathways[3]. In our present study, a comprehensive analysis of the relationship among the expression of EPHX2, prognosis in multiple cancer types and immune microenvironment was performed.

EPHX2 encodes soluble epoxide hydrolase (sEH)[4], which is an important enzyme in the degradation of endogenous lipid epoxide, especially the inactivation of epoxyeicosatrienoic acids (EETs)[5]. Dysregulation of EPHX2 contributes to the pathogenesis of several diseases, such as prostate, liver and kidney cancers[6-8], atherosclerosis[9], Parkinson's disease[10], Alzheimer's disease[11], hypertension [12] and familial hypercholesterolemia[13]. In contrast, most studies of EPHX2 in cancer

Page 2/21 
have thus far been limited to specific tumor types and there has been no report on the association studies between EPHX2 and pan-cancer. Generically, EPHX2 plays a crucial role in both the tumor progression and the immune system. Few studies have, notwithstanding, focused on the value of immunotherapy of EPHX2 in human cancer

Accordingly, in this study, we showed the expression of EPHX2 in 33 different cancers and analyzed the association between EPHX2 expression and the prognosis of patients with various types of cancer using multiple databases, including Oncomine, TIMER2, UALCAN, GEPIA2, PrognoScan, HPA and Kaplan-Meier Plotter databases. We also explored the potential relationship between EPHX2 expression and many important immunomodulators, MSI, TMB and the levels of immune infiltration in pancancer. Furthermore, we performed EPHX2-based gene set enrichment analysis (GSEA) to investigate the biological functions of EPHX2 in tumors. Additionally, the correlations between the EPHX2 expression and ICB response were explored. Taken together, this study evaluated the prognostic and immunotherapeutic value of EPHX2 in pan-cancer, providing important evidence for the function of EPHX2 and its role in clinical treatment. Notwithstanding, in consideration of the employment of bioinformatics methods, further studies are required to validate the findings.

\section{Results}

\section{The EPHX2 mRNA and protein expression levels in pan-cancer}

We first analyzed EPHX2 mRNA levels in Oncomine database to systematically interrogate the relative EPHX2 expression in different cancer types. The results indicated that EPHX2 showed generally lower expression in bladder, breast, cervical, colorectal, esophageal, head and neck, kidney, liver, lung, ovarian, and pancreatic cancers, as well as melanoma and sarcoma compared with the normal groups. Meanwhile, substantially upregulated EPHX2 expression was demonstrated in only one lung cancer dataset, one myeloma dataset and two lymphoma datasets (Fig. 1A). The details of EPHX2 expression in the above cancers were summarized in Supplementary Table 1.

To further assess the EPHX2 expression of tumor and normal tissues in human cancers, we applied the TIMER2 approach to analyse EPHX2 expression using the RNA-seq data of multiple malignancies in TCGA. As shown in Fig. 1B, EPHX2 expression was significantly down-regulated in multiple cancer types, such as BLCA, BRCA, CHOL, COAD, HNSC, KICH, KIRC, KIRP, LIHC, LUAD, LUSC, PRAD, READ, STAD, THCA and UCEC. Compared with normal tissue samples from GTEX, further validation results indicated that the expression levels of EPHX2 in ACC, BRCA, CESC, GBM, LGG, OV, PAAD, TGCT and USC tumor tissues were also profoundly decreased, and the expression was significantly up-regulated only in THYM (Fig. 1C). For normal and paired tumor tissues, EPHX2 was similarly low expression in tumor tissues of BLCA, BRCA, CHOL, COAD, HNSC, KICH, KIRC, KIRP, LIHC, LUAD, LUSC, READ, THCA and UCEC (Supplementary Figure 1).

Tellingly, at the protein level, CPTAC data also presented lower expression of EPHX2 protein in breast cancer, ovarian cancer, colon cancer, clear cell RCC, UCEC and lung adenocarcinoma primary tissues compared with normal tissues (Fig. 1D, $P<0.001$ ). Moreover, we also sought to assess EPHX2 protein expression in pan-cancer levels using the Human Protein Atlas, which demonstrated several cases of prostate and hepatocellular carcinomas as well as a few cases of renal cancer showed moderate cytoplasmic positivity and remaining cancer tissues were negative or weakly stained (Supplementary Figure 2).

Furthermore, we investigated the correlation between EPHX2 expression and the tumor pathological stages via the GEPIA2 approach as well, and observed that the EPHX2 expression was significantly associated with tumor stage in six cancers, including COAD, KICH, KIRC, KIRP, LIHC and PAAD (Fig. 1E, all $P<0.05$ ), but not others (Supplementary Figure 3).

\section{The potential prognostic value of EPHX2 in various cancers}

Next, we identified the prognostic value of EPHX2 via pan-cancer analysis in different databases. As shown in Fig. 2, EPHX2 expression was significantly associated with the prognosis of 9 cancers in the Cox proportional-hazards models, including ACC, CESC, KIRC, KIRP, LGG, LIHC, MESO, PAAD and UVM. Compared with the low EPHX2 expression group, beyond LGG (OS, overall survival: $\mathrm{HR}$, hazard ratio $=1.44, P=0.037$, DSS, disease specific survival: $\mathrm{HR}=1.52, P=0.023)$, EPHX2 acted positively in the remaining eight tumour types, incl. ACC (OS: HR $=0.39, P=0.019$, DSS: HR $=0.37, P=0.019), \mathrm{CESC}(\mathrm{OS}: \mathrm{HR}=0.55, P=0.014)$, KIRC (OS: HR $=0.52, P<0.001$, DSS: HR=0.43, $P<0.001)$, KIRP (DSS: HR=0.41, $P=0.028)$, LIHC(OS: HR=0.62, $P=0.007)$, 
MESO (OS: HR $=0.45, P=0.001$, DSS: HR $=0.50, P=0.023)$, PAAD (OS: $\mathrm{HR}=0.64, P=0.034$, DSS: $\mathrm{HR}=0.60, P=0.033)$ and UVM (OS: HR $=0.28, P=0.008$, DSS: HR $=0.27, P=0.01$ ). Simultaneously, no significant impact of EPHX2 expression on cancer prognosis was observed in other cancer types. Collectively, these results demonstrated that high-expression of EPHX2 was associated with longer survival time in ACC, CESC, KIRC, KIRP, LIHC, MESO, PAAD and UVM patients, while shorter survival time in LGG patients.

In light of these findings, we next sought to evaluate the prognostic potential of EPHX2 in different tumors using the KaplanMeier Plotter tool and data derived from the combination of the EGA, GEO and TCGA databases. Fig. 3 revealed that, in addition to its protective in CESC (OS: HR $=0.42, P=0.00025), \mathrm{KIRC}(\mathrm{OS}: \mathrm{HR}=0.44, P=4.5 \mathrm{e}-06), \mathrm{KIRP}(\mathrm{OS}: \mathrm{HR}=0.42, P=0.003)$, LIHC (OS: HR $=0.57, P=0.0013$ ) and PAAD (OS: HR $=0.53, P=0.0041)$, the EPHX2 gene also acted protectively against HNSC (OS: HR $=0.61, P=0.0048)$ and LUAD (OS: HR $=0.59, P=0.0034)$.

Finally, we evaluated the relationship between EPHX2 expression and prognosis of each cancer by PrognoScan database, which mainly extracted data from the GEO database. Detailed outcomes were summarized in Fig. 4. Our results indicated that EPHX2 played an oncogenic role in Blood cancer (OS, EFS, event free survival), and it played a protective role in Colorectal cancer (DFS, disease specific survival), Eye cancer (DMFS, distant metastasis free survival), Lung cancer (OS), Prostate cancer (OS) and Soft tissue cancer (DRFS, distant recurrence free survival). It was also worth noting that the precise role of EPHX2 in Breast cancer was controversial (such as OS of GSE9893, OS of E-TABM-158, RFS, relapse free survival of E-TABM-158 and DSS of ETABM-158: HR $>1$, Cox $P<0.05$, remarkably differed from the other datasets in Breast cancer: all HR $<1$, Cox $P<0.05)$.

Taking all the above results into consideration, both survival analysis methods, Cox proportional-hazards model and KaplanMeier plotter survival analysis, all demonstrated the significant prognostic power of EPHX2 in CESC, KIRC, KIRP, LIHC and PAAD tumors for OS or DSS. At the same time, some contradictory data related to EPHX2 expression was observed in some cancers (Table 1). These contradictory results might be due to different data collection methods, sample sizes and based on hypothetical mechanisms of different biological characteristics.

\section{The potential association between EPHX2 expression and immune related factors}

Determining the interaction of the host immune system with tumors was essential for discovering new prognostic biomarkers, decreasing drug resistance and developing novel targeted therapies[14]. It has been widely accepted that the immune infiltration in the tumor microenvironment might have a great impact on patient survival. To further explore potential correlation between EPHX2 expression and immune score, patients were divided into high and low EPHX2 expression groups using the median EPHX2 expression as the cutoff value. The results showed that EPHX2 expression was significant negatively related to immune score in BRCA, KICH, KIRC, KIRP, LIHC, MESO, OV, PRAD, SARC, TGCT, THCA and UVM, whereas positively related to immune score in DLBC, LAML, LGG, LUSC, SKCM and THYM (Fig. 5A). These results suggested that EPHX2 expression was related to abnormal immune infiltration in some cancer types and might impact the progression of cancer by affecting the regulation of immune infiltration.

In terms of immune cells infiltration, CIBERSORT algorithm was applied to analyse the relationships between EPHX2 expression and immune cell infiltrates in the tumor microenvironment. Our data also presented that the level of immune cell infiltration was significantly correlated with EPHX2 expression in most cancer types (Fig. 5B). Interestingly, EPHX2 expression showed significant negative correlation with M0 macrophage, M1 macrophage, M2 macrophage, activated NK cell and activated CD4+ memory $\mathrm{T}$ cell and positive correlation with naive CD4+ T cell, regulatory $T$ cell and follicular helper T cell in THYM. Whereas in TGCT, EPHX2 expression showed significant positive correlation with M2 macrophage and negative correlation with follicular helper $T$ cell. Furthermore, the associations between EPHX2 expression and immunomodulators were investigated $(P<0.01$ and $|\mathrm{R}|>0.5)$. As shown in Fig. 6, 24 immune inhibitors were analyzed. The EPHX2 expression showed a negative correlation with IL10 and TGFB1 in SARC, TGFB1 in MESO, and LAG3 in UVM. The correlation analysis of 45 immune stimulators (Fig. 7) revealed that EPHX2 expression was negatively correlated with ULBP1 and TNFRSF8 in UVM, CD276 in PAAD and CD276 in SARC. Strikingly, as shown in Fig. 8, a significant negative correlation was found between the expression of EPHX2 and B2M, HLA-DOB and TAP1 in UVM separately. 
Given the strong correlation of EPHX2 with PAAD, SARC, TGCT, THYM, and UVM, GSEA was performed to investigate potential several immune pathways related to EPHX2 signaling involved in these cancers (Fig. 9). Together these data indicated that EPHX2 expression was positively correlated with primary immunodeficiency pathway in PAAD. Contrasting results were found for cytokine-cytokine receptor interaction pathway in THYM and for chemokine signaling pathway and cytokine-cytokine receptor interaction pathway in UVM. Additionally, the correlation between EPHX2 and immune checkpoint blockade (TMB and MSI) was explored further. We observed that EPHX2 expression tended to have positive associations with TMB in LAML and KIRP, while inverse correlations were observed in SKCM, KIRC, PCPG, OV, BLCA, LUAD, PRAD, SARC, PAAD, BRCA, ACC, THYM and DLBC (Fig. 10A). For MSI, negative correlations with COAD, LAML and SARC were determined (Fig. 10B).

\section{The immunotherapy response prediction of EPHX2}

Cancer immunotherapy, based on harnessing patients' own immune system to fight cancer, is a promising class of treatments after surgery, radiation, and chemotherapy[15]. ICB has revolutionized the cancer therapy. Here, based on the expression profile data of TCGA, the ICB responses of high and low EPHX2 expression groups were predicted by TIDE algorithm. In 20 TCGA tumor types (ACC, BRCA, CESC, COAD, ESCA, HNSC, KIRC, LGG, LIHC, LUAD, MESO, PAAD, PRAD, READ, SARC, THCA, THYM, UCEC, LAML and TGCT), patients with high EPHX2 expression achieved a lower prediction of TIDE score compared with the low EPHX2 group (Wilcox.Tests, $P<0.05$, Fig. 11A-R). Yet, opposite results were obtained in LAML and TGCT (Wilcox.Tests, $P<0.01$, Fig. 11S-T). There were no significant differences between groups in TCGA other tumor types. Since patients with higher TIDE scores were significantly more likely to have a higher opportunity of antitumor immune escape and showed a lower response rate of ICB treatment[16], implying that patients with low-risk score appeared to be more sensitive to treated with ICB.

\section{Discussion}

Emerging publications have reported the connections between EPHX2 and clinical disorders, especially with neoplastic diseases[7, 8, 17-23]. It remains to be answered whether EPHX2 plays a role in the pathogenesis of different tumors by similar mechanisms. Through an extensive literature search, we failed to retrieve any publications for pan-cancer analysis from the perspective of the overall tumor. In this study, the purpose was to gain a greater understanding of the relationship between EPHX2 and prognosis in 33 human cancers, as well as the underlying mechanisms of the potentially immune-related associations.

We first demonstrated the prognostic value of EPHX2 in pan-cancer and we performed the analysis based on the human disease data sets. In accordance with our research, EPHX2 was generally downregulated in most of the cancer types shown in Oncomine, TIMER and CPTAC dataset, suggesting that EPHX2 might play a tumor suppressive role. Additionally, we found that EPHX2 expression was significantly correlated to tumour stages in COAD, KICH, KIRC, KIRP, LIHC and PAAD. Interestingly, we determined the prognostic value of EPHX2 expression in a variety of tumors by crossing databases, especially the consistent prognostic value in CESC, KIRC, KIRP, LIHC and PAAD. Likewise, several previous studies identified it as protective prognostic factors of oncological outcomes (e.g. PRAD[7] and HCC[8]). Even so, there was very limited information on the prognostic value of EPHX2 in other solid tumor types. The role of EPHX2 in breast cancer was controversial in the PrognoScan database. Hence, much larger sample sizes would be required to confirm the role of EPHX2 in the different types of Breast cancer prognosis and survival. Collectively, these findings strongly demonstrated that EPHX2 could serve as a prognostic biomarker in pan-cancer. Considering the low protein expression of EPHX2, we still did not recommend its use as a molecular diagnostic biomarker to identify tumors.

Synthesizing analysis across studies of EPHX2 expression differences between tumour and normal tissues, we also observed the immunotherapeutic potential of EPHX2 in a variety of tumor types. In order to achieve further insight into the potential immune value of EPHX2, we estimated the immune score from the cancer patients of TCGA cohort using the ESTIMATE algorithm and found that low EPHX2 expression was correlated with higher scores of immune infiltrations in most tumors. Also, we employed CIBERSORT to examine the relative proportions of infiltrating immune cell types in 33 cancer types. Of note, low EPHX2 expression was linked to significant infiltration of gamma delta T cells, activated memory CD4+ T cell and M0 Macrophage, respectively. Tumor-associated M0 Macrophage was generally believed to be pro-tumoral in many tumour types[24, 25]. Conclusions from the diverse studies nonetheless have been inconsistent regarding the infiltration of activated memory CD4+ T cell $[24,25]$. Compared to gamma delta T cells in the peripheral blood, tumor infiltrating gamma delta $T$ cells were known to

Page 5/21 
mediate robust anti-tumor activity[26]. These suggested that EPHX2-associated immune infiltrations in different tumors might have complex interplay affecting tumor development and progression and further demonstrated that expression changes of EPHX2 could impact immune cell infiltrations existed in the tumor microenvironment. This study now elucidates for the first time, to our knowledge, the relationships between EPHX2 and tumor immune. Therefore, our results also require additional validation by both in vivo and in vitro experiments.

Combining the results from GSEA enrichment analysis, EPHX2 in UVM might pass through activating cytokine-cytokine receptor interaction pathway and chemokine signaling pathway to promote tumorigenesis by interactions with various significantly related immune factors such as LAG3, ULBP1 and TNFRSF8, and MHC molecules such as B2M, HLA-DOB, TAP1, etc. Furthermore, in this study, both biomarkers (TMB and MSI) for immunotherapy showed a significant association with EPHX2 in certain cancers. Generally speaking, the higher the TMB value was, the more somatic mutation-related neoantigens were produced[27]. By contrast, MSI was defined as hypermutator phenotypes caused by DNA mismatch repair deficiency and was a potential predictive marker for immunotherapy[28]. In SARC, EPHX2 was inversely correlated with TMB and MSI, while the opposite was the case in LAML, which suggested that EPHX2 might have indirect effects on the immunotherapy response of SARC and LAML.

In closing, we demonstrated the correlations between EPHX2 and immunotherapy response. Taken together with these data, our findings indicated that high EPHX2 expression subtype patients might be more responsive to ICB in the majority of TCGA tumors. ICB therapy has emerged as one of the most promising approaches to activate antitumor immune response and has made great success in treatment of various cancers, which could produce durable responses and prolong survival. Despite the considerable therapeutic potential, all patients did not derive benefit equally well from this approach. Accumulating evidence has indicated that the efficacy of ICB mainly depends on the robust anti-tumor immunity responses, which were commonly suggested to be compromised in most tumors. Therefore, the conclusions summarized above should be verified in future clinical trials. This is the first study, to the best of our knowledge, to comprehensively report on the value of EPHX2 in multiple cancers (33 types). This study provides new insights into the role of EPHX2 in cancer immunotherapy and reveals the associations between EPHX2 and important immunological indicators (immune cell infiltrations, immunomodulators, and immune biomarkers), which might be useful to understand the underlying mechanism by linking EPHX2 and immunotherapy. These findings underscore the immunological role of EPHX2 in specific cancers, which will become an effective mean to target them, although not all tumors presented the association between EPHX2 and TME. None-the-less, in view of the bioinformatics methods adopted in the present study, these findings are preliminary in nature. Thus, ultimately, extensive functional in vitro and in vivo work is warranted.

\section{Conclusions}

Overall, this study investigated the first comprehensive immunotherapy value of EPHX2 in 33 human cancers. We believe that these findings will set the stage for prospective functional experiments and ultimately may affect the clinical environment.

\section{Materials And Methods}

\section{Gene expression analysis}

The Oncomine database (www.oncomine.org) was employed to analyze the mRNA expression levels of the EPHX2 gene in various types of cancers[29]. The threshold was defined as: $P$-value of 0.0001 , fold change of 2.0 and gene rank of top $10 \%$. We further assessed the differential expression levels of EPHX2 between tumors or specific tumor subtypes and adjacent normal tissues in the TCGA project by using TIMER database (http://cistrome.org/TIMER/)[14]. For certain cancer types without normal tissues or with highly limited normal tissues [e.g., TCGA-ACC, TCGA-BRCA, etc.], we further used the UCSC XENA (https://xenabrowser.net/datapages/) RNAseq data in TPM format of TCGA and GTEx through the Toil[30] process and performed log2 transformation to compare the expression of samples to obtain box plots of the EPHX2 mRNA expression difference between the these tumor tissues and the corresponding normal tissues.

In addition, the analysis of protein expression levels of EPHX2 was performed by UALCAN portal (http://ualcan.path.uab.edu/analysis-prot.html) based on the CPTAC database[31]. Here, we separately explored the total protein 
expression levels of EPHX2 in six available tumor data sets (primary tumor and normal tissues), such as breast cancer, ovarian cancer, colon cancer, clear cell RCC, UCEC, and lung adenocarcinoma.

Moreover, the "Pathological Stage Plot" module of GEPIA2 web server (http://gepia2.cancer-pku.cn/\#analysis)[32] was used to identify violin plots of EPHX2 expression across various pathological stages (stage I, stage II, stage III, and stage IV) of all tumors available on the TCGA database.

\section{Survival prognosis analysis}

The Cox proportional hazards models were carried out to assess the prognostic value of the EPHX2 gene on survival in tumors[33]. Kaplan-Meier Plotter, a powerful in silico online tool (http://kmplot.com/analysis/) that enables users to predict patient survival across various cancer types of TCGA based on large sample datasets[34]. PrognoScan database (http://www.prognoscan.org/) is a large collection of publicly available cancer microarray datasets and a tool for assessing the biological relationships between gene expression and prognosis, such as OS and DFS[35].

\section{Analysis of Potential Association Between EPHX2 Expression and Immune-Related Factors}

The immune score of each sample was inferred according to the ESTIMATE algorithm [36]. Afterwards, the CIBERSORT algorithm was utilized to assess the relative fractions of 22 infiltrating immune cell types in each tumor sample[37]. Previous studies have shown that TMB and MSI were closely linked to immune responses. Therefore, the study also examined the correlations between EPHX2 expression and these indicators. TMB was defined as the total number of somatic, coding, base substitutions, and indel mutations per $\mathrm{Mb}$ of genome examined. To calculate the TMB per megabase, the total number of mutations counted was divided by the $38 \mathrm{Mb}$ exome size[38]. The MSI score of each sample from TCGA database was accessed using the previously published studies[39]. Besides, the potential relationships between EPHX2 expression and immunomodulators (immunosuppressants, immunostimulants and MHC molecules) were explored by TISIDB website (http://cis.hku.hk/TISIDB/index.php)[40]. The top 4 strongest associations were highlighted on the figures. Finally, Gene set enrichment analysis (GSEA) was conducted in the highexpression and the low-expression groups compared with the median level of EPHX2 expression respectively to further investigate the top differentially regulated biological signaling pathways. The top 5 significant pathways $(P<0.05)$ in two groups with the highest normalized enrichment score were considered and exhibited on the graphs.

\section{The prediction of ICB response}

Potential ICB response was predicted with TIDE algorithm[41]. TIDE integrated a set of gene expression markers to evaluate two different mechanisms of tumour immune evasion: dysfunction of tumor-infiltrating cytotoxic T-lymphocytes (CTL) and the rejection to CTL by immunosuppressive factors. Higher scoring represented the worse efficacy of ICB therapy and shorter survival after ICB treatment.

\section{Statistical analysis}

Cancer patients were separated into high and low EPHX2 expression groups based on the median value of EPHX2 expression. Survival differences were described as Kaplan-Meier curves with median comparison by log-rank tests and hazard ratios from Cox proportional-hazards models. The correlations between EPHX2 expression and checkpoint-related genes were performed by Pearson's correlation analysis. Differences in continuous variables were compared using the Wilcoxon signed-rank test. Multiple hypothesis testing was adjusted by the Benjamini-Hochberg method [42]. All statistical analysis was carried out in $\mathrm{R}$ version 4.0.2 with $P$ values of $\leq 0.05$ considered significant.

\section{Abbreviations}

TME, Tumor immune microenvironment, TMB, Tumor mutational burden, MSI, Microsatellite instability, ICB, Immune checkpoint blocker, TIDE, Tumor immune dysfunction and exclusion, TCGA, The Cancer Genome Atlas, sEH, soluble Epoxide Hydrolase, EETs, Epoxyeicosatrienoic acids, GSEA, Gene set enrichment analysis, PAAD, Pancreatic adenocarcinoma, THYM, Thymoma, UVM, Uveal melanoma, ACC, adrenocortical carcinoma, BRCA, breast invasive carcinoma, clear cell RCC, Renal cell carcinoma, CNS, 
central nervous system, BLCA, bladder urothelial carcinoma, CESC, cervical squamous cell carcinoma and endocervical adenocarcinoma, $\mathrm{CHOL}$, cholangiocarcinoma, COAD, colon adenocarcinoma, DLBC, lymphoid neoplasm diffuse large B-cell lymphoma, ESCA, esophageal carcinoma, GBM, glioblastoma multiforme, HNSC, head and neck squamous cell carcinoma, HNSC-HPVpos, Head and neck cancer-HPV positive, HNSC-HPVneg, Head and neck cancer-HPV negative, KICH, kidney chromophobe, KIRC, kidney renal clear cell carcinoma, KIRP, kidney renal papillary cell carcinoma, LAML, acute myeloid leukemia, LGG, brain lower grade glioma, LIHC, liver hepatocellular carcinoma, LUAD, lung adenocarcinoma, LUSC, lung squamous cell carcinoma, MESO, mesothelioma, OV, Ovarian serous cystadenocarcinoma, PCPG, pheochromocytoma and paraganglioma, PRAD, Prostate adenocarcinoma, READ, Rectum adenocarcinoma, SARC, sarcoma, SKCM, skin cutaneous melanoma, STAD, stomach adenocarcinoma, TGCT, Testicular germ cell tumors, THCA, Thyroid carcinoma, UCS, Uterine carcinosarcoma, OS, Overall survival, DSS, Disease specific survival, HR, Hazard ratio, RFS, Relapse free survival, EFS, Event free survival, DFS, Disease specific survival, DMFS, Distant metastasis free survival, DRFS, Distant recurrence free survival, CTL, Cytotoxic Tlymphocyte.

\section{Declarations}

\section{Acknowledgements}

We would like to sincerely thank the various contributors to the open-access databases, including Oncomine, TIMER2, TCGA, GTEx, UALCAN, CPTAC, GEPIA2, PrognoScan, TISIDB, HPA, GEO and Kaplan-Meier Plotter, that our data were downloaded from.

\section{Authors' contributions}

WH: conceived the idea, designed the analyses, planned this project, interpreted the results and drafted the manuscript. QZ: conceived the idea, designed the analyses, planned this project, interpreted the results and drafted the manuscript. JX: analyzed the data and prepared supplementary material. HL: acquired the data and prepared figures 1-2 and table 1. LZ: acquired the data and prepared figures 3-5. SH: acquired the data and prepared figures 6-8. JL: acquired the data and prepared figures 9-11. FC: conceived the idea, designed the analyses, planned this project, interpreted the results and drafted the manuscript. All authors reviewed and critically revised the manuscript and approved the final manuscript for submission.

\section{Funding}

Our work was supported by the Natural Science Foundation of China (Grant No. 82002233) and the Natural Science Foundation of Jiangsu Province of China (Grant No. BK20180293).

\section{Availability of data and materials}

The data set provided in this study can be accessed at online repositories. The Oncomine database(www.oncomine.org), TIMER database (http://cistrome.org/TIMER/), UCSC XENA database (https://xenabrowser.net/datapages/), UALCAN portal database (http://ualcan.path.uab.edu/analysis-prot.html), TCGA database (https://tcga-data.nci.nih.gov/tcga), GEPIA2 database(http://gepia2.cancer-pku.cn/\#analysis), Kaplan-Meier Plotter database(http://kmplot.com/analysis/), PrognoScan database (http://www.prognoscan.org/), TISIDB database (http://cis.hku.hk/TISIDB/index.php), GEO database (https://www.ncbi.nlm.nih.gov/geo), GTEx database(www.gtexportal.org/home/) and HPA database(https://www.proteinatlas.org/) are publicly available databases.

\section{Ethics approval and consent to participate}

All procedures were performed in accordance with relevant guidelines.

\section{Consent for publication}

Not applicable.

\section{Competing interests}


All authors declared no potential conflict of interest.

\section{Supplementary material}

\section{Author details}

${ }^{1}$ Department of Joint Surgery, Ganzhou People's Hospital, Ganzhou 341000, Jiangxi, China. ${ }^{2}$ Department of Laboratory Medicine, First Affiliated Hospital of Gannan Medical University, Ganzhou 341000, Jiangxi, China. ${ }^{3}$ Department of Orthopaedic Surgery, Sun Yat-Sen Memorial Hospital, Sun Yat-Sen University, Guangzhou 510120, Guangdong, China. ${ }^{4}$ Department of Clinical College, Xiangtan Medicine \& Health Vocational College, Xiangtan 411104, Hunan, China. ${ }^{5}$ Department of Radiotherapy, The Fourth Hospital of Hebei Medical University, Shijiazhuang 050035, Hebei, China. ${ }^{6}$ Department of Emergency, First Affiliated Hospital of Gannan Medical University, Ganzhou 341000, Jiangxi, China. ${ }^{7}$ Department of Medical Technology, Gannan Healthcare Vocational College, Ganzhou 341000, Jiangxi, China. ${ }^{8}$ Basic Medical Laboratory, Institute of Clinical Laboratory, Jinling hospital, Nanjing 210002, Jiangsu, China.

\section{References}

1. Bray F, Ferlay J, Soerjomataram I, Siegel RL, Torre LA, Jemal A. Global cancer statistics 2018: GLOBOCAN estimates of incidence and mortality worldwide for 36 cancers in 185 countries. CA: a cancer journal for clinicians. 2018, 68(6):394-424.

2. Ribas A, Wolchok JD. Cancer immunotherapy using checkpoint blockade. Science. 2018, 359(6382):1350-5.

3. Blum A, Wang P, Zenklusen JC. SnapShot: TCGA-Analyzed Tumors. Cell. 2018, 173(2):530.

4. Larsson C, White I, Johansson C, Stark A, Meijer J. Localization of the human soluble epoxide hydrolase gene (EPHX2) to chromosomal region 8p21-p12. Human genetics. 1995, 95(3):356-8.

5. Spector AA. Arachidonic acid cytochrome P450 epoxygenase pathway. Journal of lipid research. 2009, 50 Suppl(Suppl):S526.

6. Enayetallah AE, French RA, Grant DF. Distribution of soluble epoxide hydrolase, cytochrome P450 2C8, 2C9 and 2J2 in human malignant neoplasms. Journal of molecular histology. 2006, 37(3-4):133-41.

7. Liu MS, Zhao H, Xu CX, Xie PB, Wang W, Yang YY, Lee WH, Jin Y, Zhou HQ. Clinical significance of EPHX2 deregulation in prostate cancer. Asian journal of andrology. 2021, 23(1):109-15.

8. Zhan K, Bai Y, Liao S, Chen H, Kuang L, Luo Q, Lv L, Qiu L, Mei Z. Identification and validation of EPHX2 as a prognostic biomarker in hepatocellular carcinoma. Mol Med Rep. 2021, 24(3).

9. Roumeliotis S, Roumeliotis A, Stamou A, Panagoutsos S, Manolopoulos VG, Tsetsos F, Georgitsi M, Liakopoulos V. Association of rs11780592 Polymorphism in the Human Soluble Epoxide Hydrolase Gene (EPHX2) with Oxidized LDL and Mortality in Patients with Diabetic Chronic Kidney Disease. Oxidative medicine and cellular longevity. 2021, 2021:8817502.

10. Oguro A, Ishihara Y, Siswanto FM, Yamazaki T, Ishida A, Imaishi H, Imaoka S. Contribution of DHA diols (19,20-DHDP) produced by cytochrome P450s and soluble epoxide hydrolase to the beneficial effects of DHA supplementation in the brains of rotenone-induced rat models of Parkinson's disease. Biochimica et biophysica acta Molecular and cell biology of lipids. 2021, 1866(2):158858.

11. Chen W, Wang M, Zhu M, Xiong W, Qin X, Zhu X. 14,15-Epoxyeicosatrienoic Acid Alleviates Pathology in a Mouse Model of Alzheimer's Disease. The Journal of neuroscience : the official journal of the Society for Neuroscience. 2020, 40(42):8188203.

12. Ma L, Zhao H, Yu M, Wen Y, Zhao T, Yan M, Liu Q, Jiang Y, Cao Y, Li P, et al. Association of Epoxide Hydrolase 2 Gene Arg287GIn with the Risk for Primary Hypertension in Chinese. International journal of hypertension. 2020, 2020:2351547.

13. Sato K, Emi M, Ezura Y, Fujita Y, Takada D, Ishigami T, Umemura S, Xin Y, Wu LL, Larrinaga-Shum S, et al. Soluble epoxide hydrolase variant (Glu287Arg) modifies plasma total cholesterol and triglyceride phenotype in familial hypercholesterolemia: intrafamilial association study in an eight-generation hyperlipidemic kindred. Journal of human genetics. 2004, 49(1):29-34. 
14. Li B, Severson E, Pignon JC, Zhao H, Li T, Novak J, Jiang P, Shen H, Aster JC, Rodig S, et al. Comprehensive analyses of tumor immunity: implications for cancer immunotherapy. Genome biology. 2016, 17(1):174.

15. Feng Z, Zhang T, Wang H, Xu B. Supramolecular catalysis and dynamic assemblies for medicine. Chemical Society reviews. 2017, 46(21):6470-9.

16. Wang Q, Li M, Yang M, Yang Y, Song F, Zhang W, Li X, Chen K. Analysis of immune-related signatures of lung adenocarcinoma identified two distinct subtypes: implications for immune checkpoint blockade therapy. Aging. 2020, 12(4):3312-39.

17. Liu J, Nie S, Gao M, Jiang Y, Wan Y, Ma X, Zhou S, Cheng W. Identification of EPHX2 and RMI2 as two novel key genes in cervical squamous cell carcinoma by an integrated bioinformatic analysis. J Cell Physiol. 2019, 234(11):21260-73.

18. Pedro NF, Biselli JM, Maniglia JV, Santi-Neto D, Pavarino É C, Goloni-Bertollo EM, Biselli-Chicote PM. Candidate Biomarkers for Oral Squamous Cell Carcinoma: Differential Expression of Oxidative Stress-Related Genes. Asian Pac J Cancer Prev. 2018, 19(5):1343-9.

19. Zhang R, Ye J, Huang H, Du X. Mining featured biomarkers associated with vascular invasion in HCC by bioinformatics analysis with TCGA RNA sequencing data. Biomedicine \& pharmacotherapy = Biomedecine \& pharmacotherapie. 2019, 118:109274.

20. Gautheron J, Jéru I. The Multifaceted Role of Epoxide Hydrolases in Human Health and Disease. Int J Mol Sci. 2020, 22(1).

21. Grigo K, Wirsing A, Lucas B, Klein-Hitpass L, Ryffel GU. HNF4 alpha orchestrates a set of 14 genes to down-regulate cell proliferation in kidney cells. Biological chemistry. 2008, 389(2):179-87.

22. Figueroa JD, Malats N, García-Closas M, Real FX, Silverman D, Kogevinas M, Chanock S, Welch R, Dosemeci M, Lan Q, et al. Bladder cancer risk and genetic variation in AKR1C3 and other metabolizing genes. Carcinogenesis. 2008, 29(10):1955-62.

23. Vainio P, Gupta S, Ketola K, Mirtti T, Mpindi JP, Kohonen P, Fey V, Perälä M, Smit F, Verhaegh G, et al. Arachidonic acid pathway members PLA2G7, HPGD, EPHX2, and CYP4F8 identified as putative novel therapeutic targets in prostate cancer. The American journal of pathology. 2011, 178(2):525-36.

24. Zhang F, Wang X, Bai Y, Hu H, Yang Y, Wang J, Tang Y, Ma H, Feng D, Li D. Development and Validation of a Hypoxia-Related Signature for Predicting Survival Outcomes in Patients With Bladder Cancer. Frontiers in genetics. 2021, 12:670384.

25. Li W, Zeng J, Luo B, Mao Y, Liang Y, Zhao W, Hu N, Chen G, Zheng X. [High expression of activated CD4(+) memory T cells and CD8(+) T cells and low expression of M0 macrophage are associated with better clinical prognosis in bladder cancer patients]. Xi bao yu fen zi mian yi xue za zhi = Chinese journal of cellular and molecular immunology. 2020, 36(2):97-103.

26. Chen H, Zou M, Teng D, Hu Y, Zhang J, He W. Profiling the pattern of the human T-cell receptor y $\delta$ complementary determinant region 3 repertoire in patients with lung carcinoma via high-throughput sequencing analysis. Cellular \& molecular immunology. 2019, 16(3):250-9.

27. Chan TA, Yarchoan M, Jaffee E, Swanton C, Quezada SA, Stenzinger A, Peters S. Development of tumor mutation burden as an immunotherapy biomarker: utility for the oncology clinic. Annals of oncology : official journal of the European Society for Medical Oncology. 2019, 30(1):44-56.

28. Yamamoto $\mathrm{H}$, Imai K. An updated review of microsatellite instability in the era of next-generation sequencing and precision medicine. Seminars in oncology. 2019, 46(3):261-70.

29. Rhodes DR, Kalyana-Sundaram S, Mahavisno V, Varambally R, Yu J, Briggs BB, Barrette TR, Anstet MJ, Kincead-Beal C, Kulkarni P, et al. Oncomine 3.0: genes, pathways, and networks in a collection of 18,000 cancer gene expression profiles. Neoplasia (New York, NY). 2007, 9(2):166-80.

30. Vivian J, Rao AA, Nothaft FA, Ketchum C, Armstrong J, Novak A, Pfeil J, Narkizian J, Deran AD, Musselman-Brown A, et al. Toil enables reproducible, open source, big biomedical data analyses. Nature biotechnology. 2017, 35(4):314-6.

31. Chen F, Chandrashekar DS, Varambally S, Creighton CJ. Pan-cancer molecular subtypes revealed by mass-spectrometrybased proteomic characterization of more than 500 human cancers. Nat Commun. 2019, 10(1):5679.

32. Tang Z, Kang B, Li C, Chen T, Zhang Z. GEPIA2: an enhanced web server for large-scale expression profiling and interactive analysis. Nucleic Acids Res. 2019, 47(W1):W556-60. 
33. Liu J, Lichtenberg T, Hoadley KA, Poisson LM, Lazar AJ, Cherniack AD, Kovatich AJ, Benz CC, Levine DA, Lee AV, et al. An Integrated TCGA Pan-Cancer Clinical Data Resource to Drive High-Quality Survival Outcome Analytics. Cell. 2018, 173(2):400-16.e411.

34. Lánczky A, Nagy Á, Bottai G, Munkácsy G, Szabó A, Santarpia L, Győrffy B. miRpower: a web-tool to validate survivalassociated miRNAs utilizing expression data from 2178 breast cancer patients. Breast cancer research and treatment. 2016, 160(3):439-46.

35. Mizuno H, Kitada K, Nakai K, Sarai A. PrognoScan: a new database for meta-analysis of the prognostic value of genes. BMC Med Genomics. 2009, 2:18.

36. Yoshihara K, Shahmoradgoli M, Martínez E, Vegesna R, Kim H, Torres-Garcia W, Treviño V, Shen H, Laird PW, Levine DA, et al. Inferring tumour purity and stromal and immune cell admixture from expression data. Nat Commun. 2013, 4:2612.

37. Newman AM, Liu CL, Green MR, Gentles AJ, Feng W, Xu Y, Hoang CD, Diehn M, Alizadeh AA. Robust enumeration of cell subsets from tissue expression profiles. Nature methods. 2015, 12(5):453-7.

38. Thorsson V, Gibbs DL, Brown SD, Wolf D, Bortone DS, Ou Yang TH, Porta-Pardo E, Gao GF, Plaisier CL, Eddy JA, et al. The Immune Landscape of Cancer. Immunity. 2019, 51(2):411-2.

39. Bonneville R, Krook MA, Kautto EA, Miya J, Wing MR, Chen HZ, Reeser JW, Yu L, Roychowdhury S. Landscape of Microsatellite Instability Across 39 Cancer Types. JCO precision oncology. 2017, 2017.

40. Ru B, Wong CN, Tong Y, Zhong JY, Zhong SSW, Wu WC, Chu KC, Wong CY, Lau CY, Chen I, et al. TISIDB: an integrated repository portal for tumor-immune system interactions. Bioinformatics. 2019, 35(20):4200-2.

41. Jiang P, Gu S, Pan D, Fu J, Sahu A, Hu X, Li Z, Traugh N, Bu X, Li B, et al. Signatures of T cell dysfunction and exclusion predict cancer immunotherapy response. Nature medicine. 2018, 24(10):1550-8.

42. Love MI, Huber W, Anders S. Moderated estimation of fold change and dispersion for RNA-seq data with DESeq2. Genome biology. 2014, 15(12):550.

\section{Tables}

Table 1. The association between EPHX2 gene high expression and pan-cancer in different database.

\begin{tabular}{|c|c|c|c|c|c|c|c|c|c|c|}
\hline \multirow[t]{2}{*}{ Role } & \multicolumn{2}{|l|}{$\begin{array}{c}\text { TCGA } \\
(\text { COX })\end{array}$} & \multirow{2}{*}{$\begin{array}{l}\begin{array}{l}\text { Kaplan- } \\
\text { Meier } \\
\text { plotter }\end{array} \\
\text { OS }\end{array}$} & \multicolumn{7}{|c|}{ PrognoScan } \\
\hline & os & DSS & & os & DSS & DFS & RFS & EFS & DMFS & DRFS \\
\hline Protective & $\begin{array}{l}\text { ACC, } \\
\text { CESC, } \\
\text { KIRC, } \\
\text { LIHC, } \\
\text { MESO, } \\
\text { PAAD, } \\
\text { UVM }\end{array}$ & $\begin{array}{l}\text { ACC, } \\
\text { KIRC, } \\
\text { KIRP, } \\
\text { MESO, } \\
\text { PAAD, } \\
\text { UVM }\end{array}$ & $\begin{array}{l}\text { CESC, } \\
\text { KIRC, } \\
\text { KIRP, } \\
\text { LIHC, } \\
\text { PAAD, } \\
\text { HNSC, } \\
\text { LUAD }\end{array}$ & $\begin{array}{l}\text { Breast } \\
\text { cancer, } \\
\text { Lung } \\
\text { cancer, } \\
\text { Prostate } \\
\text { cancer }\end{array}$ & $\begin{array}{l}\text { Breast } \\
\text { cancer }\end{array}$ & $\begin{array}{l}\text { Breast } \\
\text { cancer, } \\
\text { Colorectal } \\
\text { cancer }\end{array}$ & $\begin{array}{l}\text { Breast } \\
\text { cancer }\end{array}$ & & $\begin{array}{l}\text { Breast } \\
\text { cancer, } \\
\text { Eye } \\
\text { cancer }\end{array}$ & $\begin{array}{l}\text { Soft } \\
\text { tissue } \\
\text { cancer }\end{array}$ \\
\hline Detrimental & LGG & LGG & & $\begin{array}{l}\text { Blood } \\
\text { cancer, } \\
\text { Breast } \\
\text { cancer }\end{array}$ & $\begin{array}{l}\text { Breast } \\
\text { cancer }\end{array}$ & & $\begin{array}{l}\text { Breast } \\
\text { cancer }\end{array}$ & $\begin{array}{l}\text { Blood } \\
\text { cancer }\end{array}$ & & \\
\hline
\end{tabular}

Significance of bold indicated that the high expression level of EPHX2 was significantly correlated with an improved OS or DSS for these cancers, and the correlation was consistent across different databases.

\section{Figures}




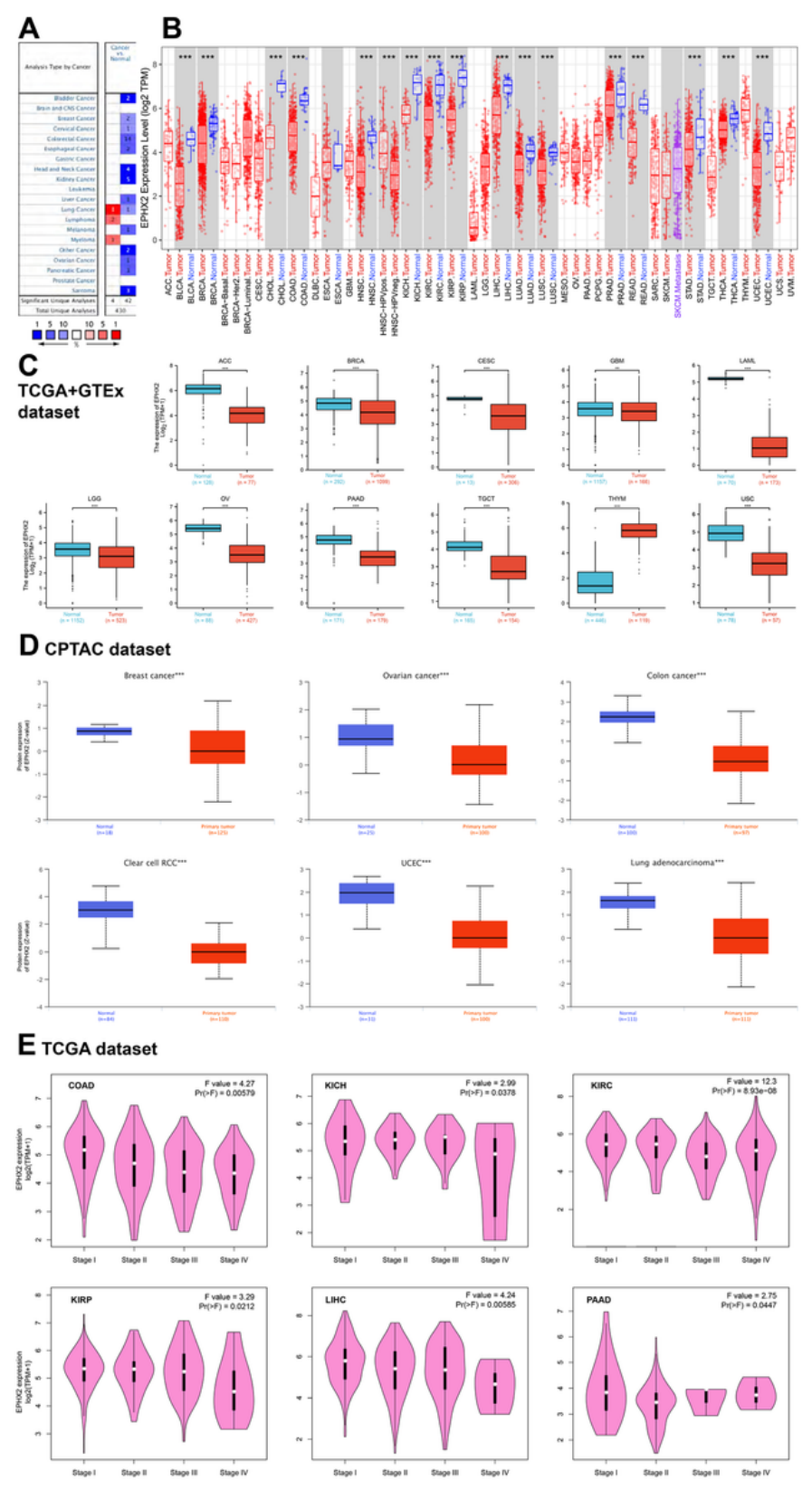

Figure 1

Expression levels of EPHX2 in different tumor types and pathological stages. A The EPHX2 expression levels in different normal tissues and normal tissues from Oncomine database ( $P$-value: 0.0001 , fold change: 2.0 , gene rank: top 10\%). B The EPHX2 expression status in diverse cancers or specific cancer subtypes using TIMER2C database $\left({ }^{\star} P<0.05,{ }^{\star} P<0.01,{ }^{\star * \star} P<0.001\right)$. C Box plot analyzed the EPHX2 expression in ACC, USC, BRCA, CESC, GBM, LAML, LGG, OV, PAAD, TGCT and THYM of TCGA project and normal tissues based on GTEx database ( ${ }^{\star \star} P<0.01$, $\left.{ }^{\star \star \star} P<0.001\right)$. D The EPHX2 total-Protein expression levels of cancer and normal tissues in breast cancer, ovarian cancer, colon cancer, clear cell RCC, UCEC and lung adenocarcinoma were analyzed based on the CPTAC dataset ( $\star \star \star P<0.001)$. E Based on the TCGA data, the expression levels of the EPHX2 gene were analyzed by the main pathological stages (stage I, stage II, stage III, and stage IV) of COAD, KICH, KIRC, KIRP, LIHC and PAAD. Log2 (TPM+1) was applied for log-scale. 

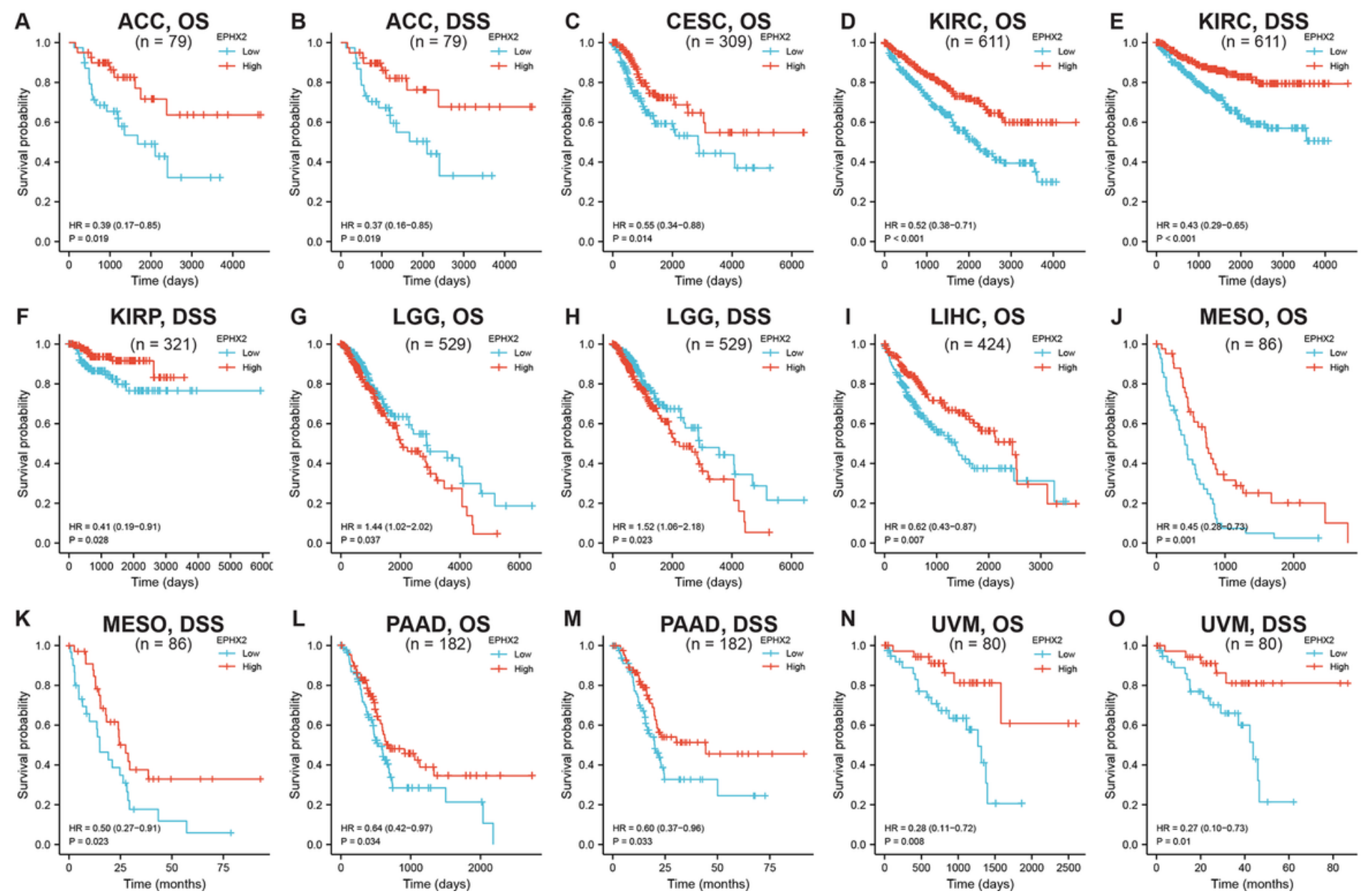

Figure 2

Kaplan-Meier survival curves for pan-cancer with high and low EPHX2 expression. 

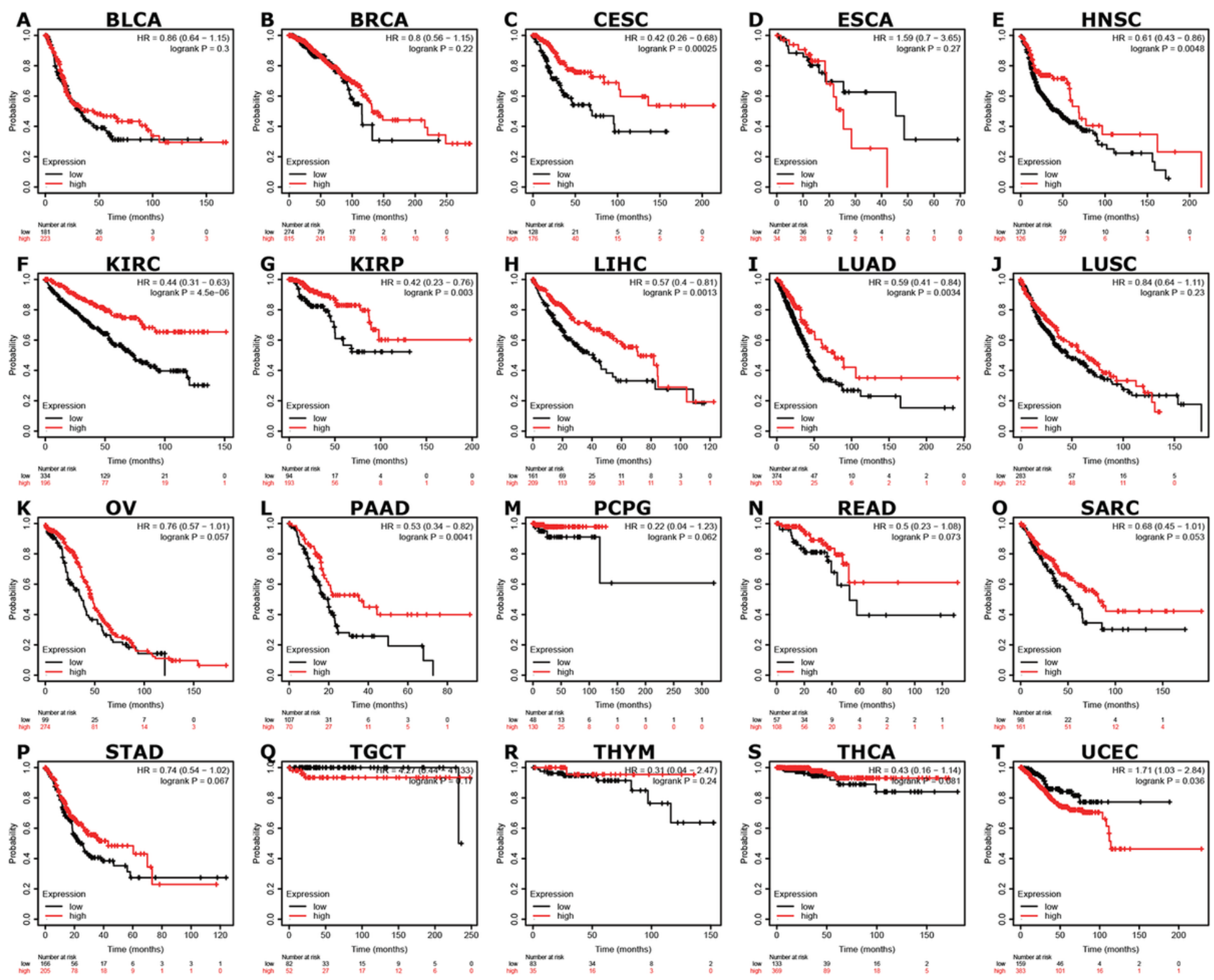

Figure 3

OS curves of high and low EPHX2 expression in various cancers in Kaplan-Meier Plotter database. A EPHX2 in BLCA. B EPHX2 in BRCA. C EPHX2 in CESC. D EPHX2 in ESCA. E EPHX2 in HNSC. F EPHX2 in KIRC. G EPHX2 in KIRP. HEPHX2 in LIHC. I EPHX2 in LUAD. J EPHX2 in LUSC. KEPHX2 in OV. L EPHX2 in PAAD. M EPHX2 in PCPG. NEPHX2 in READ. 0 EPHX2 in SARC. P EPHX2 in STAD. Q EPHX2 in TGCT. R EPHX2 in THYM. S EPHX2 in THCA. T EPHX2 in UCEC. 


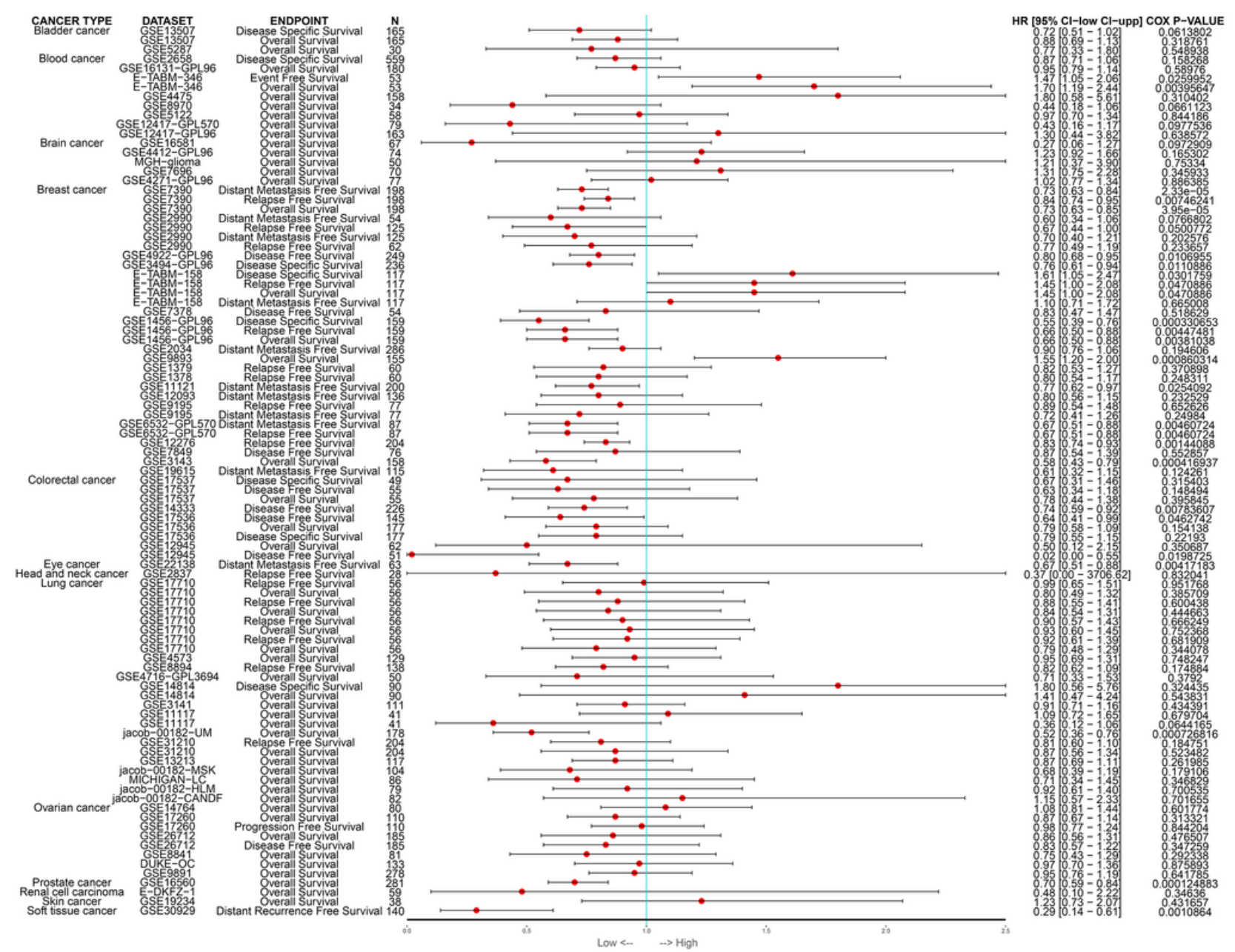

Figure 4

Pan-cancer prognostic analysis of EPHX2 expression in different datasets of cancers in PrognoScan. The red circle represents the hazard ratio. 

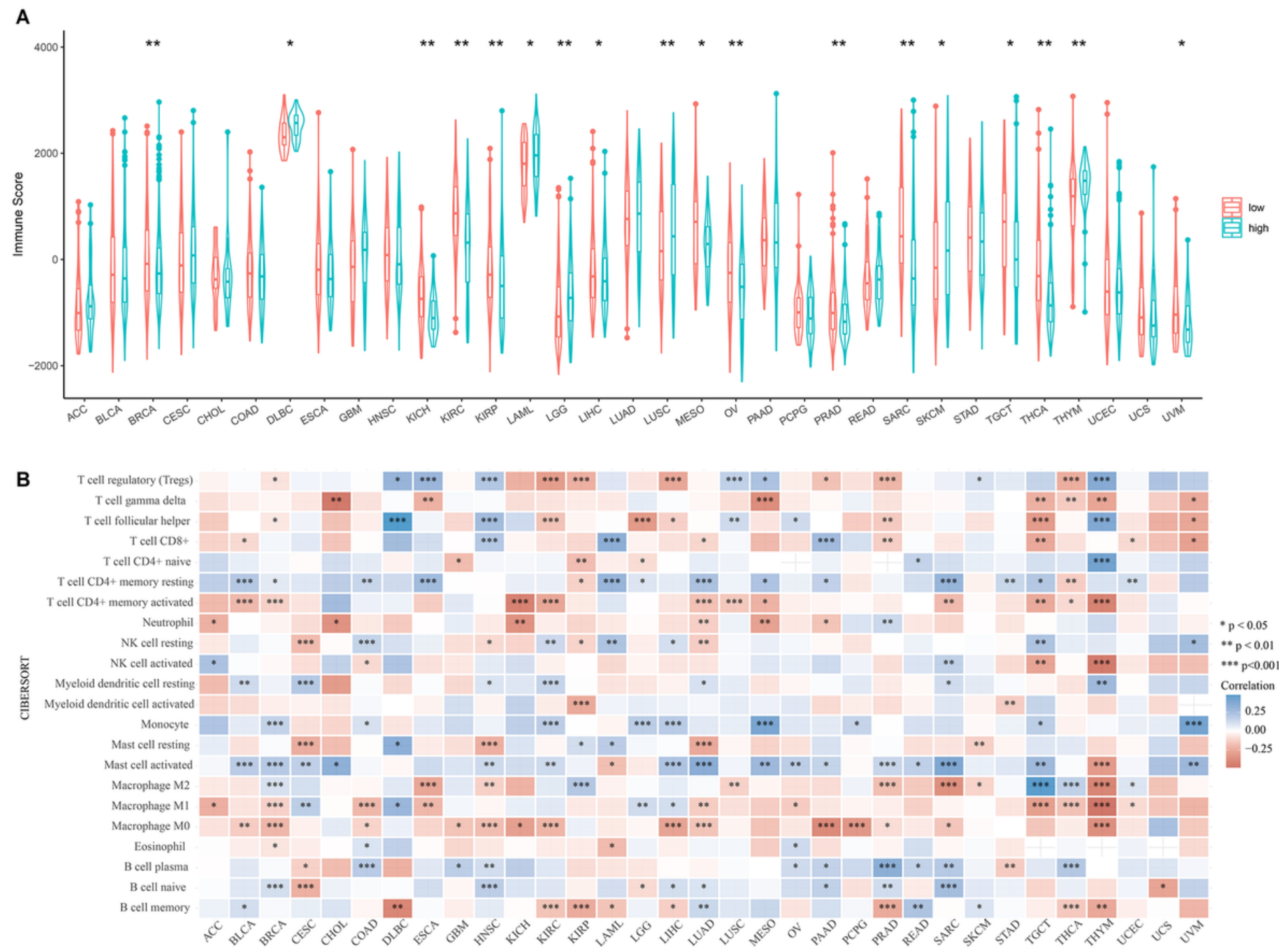

\section{Figure 5}

The relationship between the EPHX2 expression and TME factors. A Correlation between the EPHX2 expression and immune score of 33 different cancer types. B Correlation between the EPHX2 expression and immune infiltrating subtypes of 33 cancer types. Blue represents positive correlations and red represents negative correlations $\left({ }^{\star} P<0.05,{ }^{\star \star} P<0.01,{ }^{\star \star \star} P<0.001\right)$. 

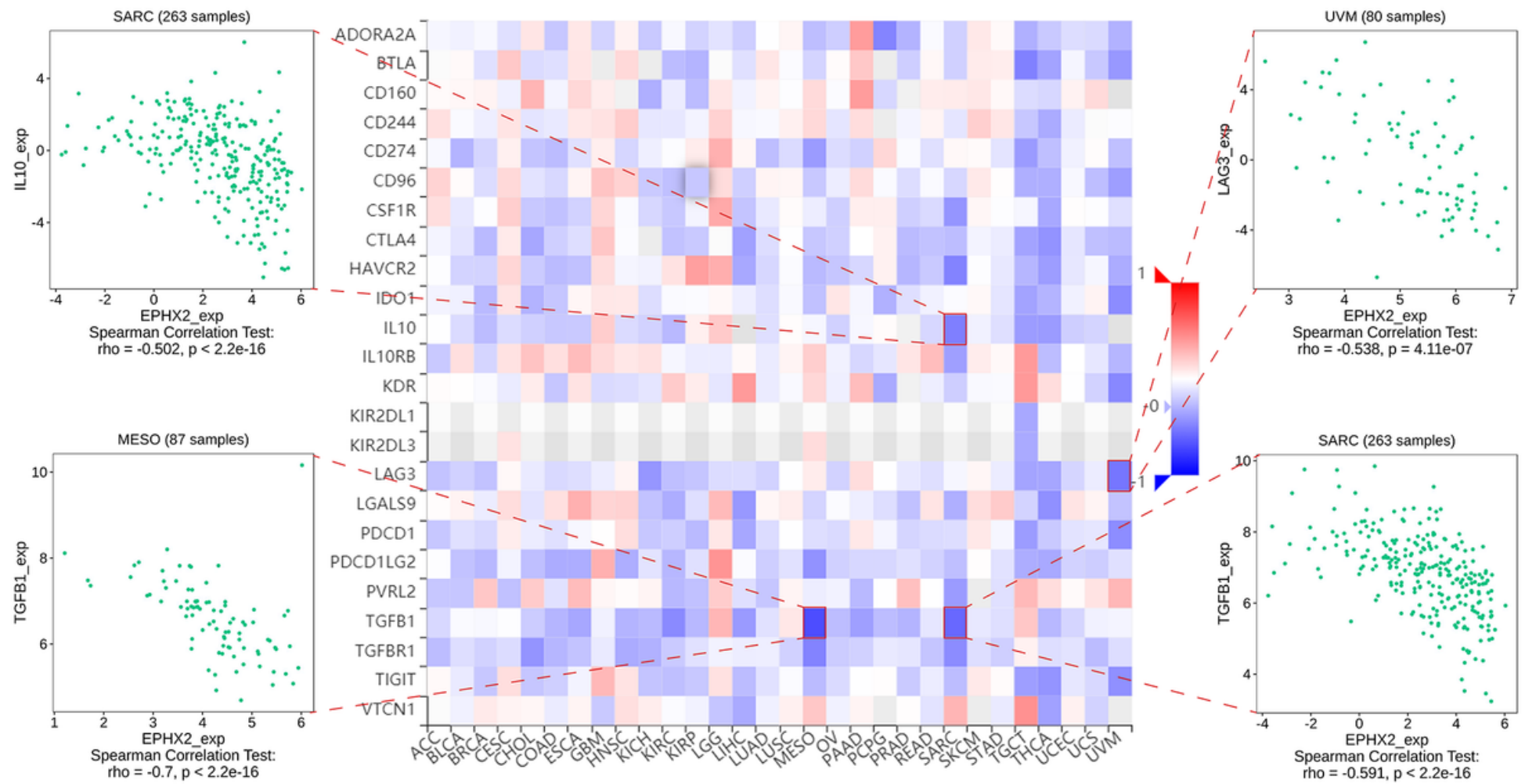

Figure 6

The correlation between the EPHX2 expression and immune inhibitors. Red indicates positive correlation and blue indicates negative correlation. The first 4 strongest associations are shown by dot plots.
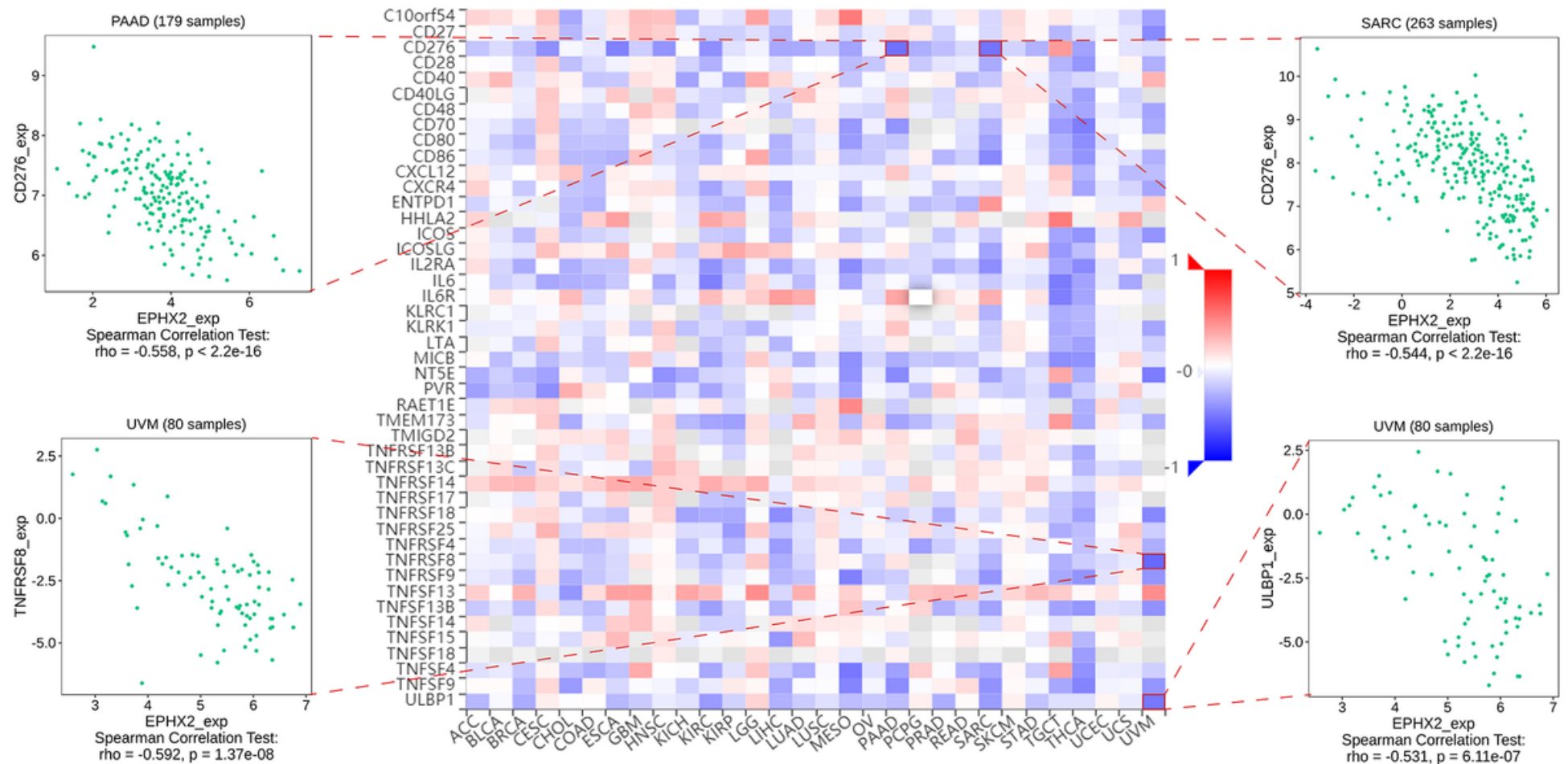

Figure 7

The correlation between the EPHX2 expression and immune stimulators. Red indicates positive correlation and blue indicates negative correlation. The first 4 strongest associations are shown by dot plots. 


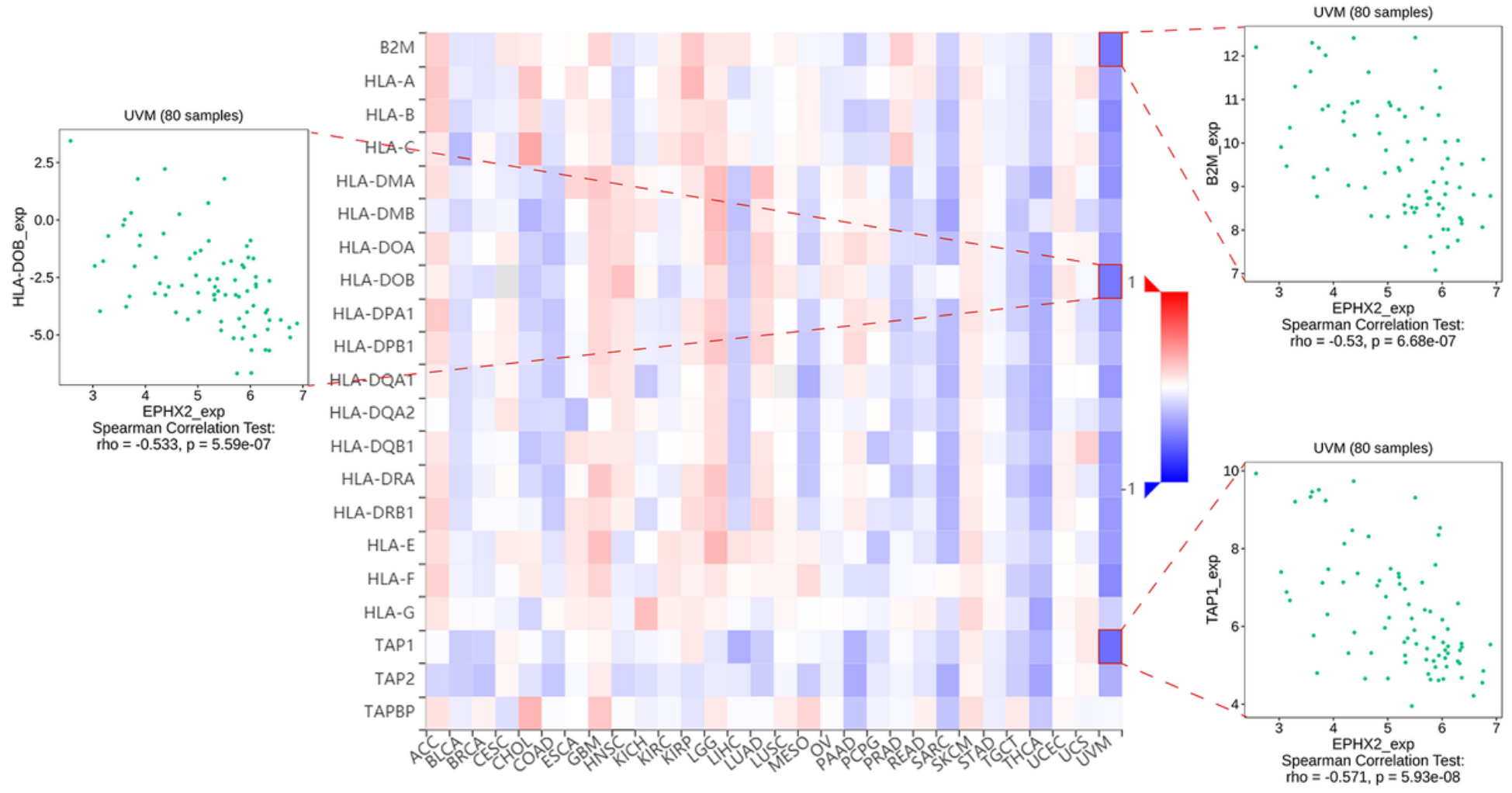

\section{Figure 8}

The correlation between the EPHX2 expression and MHC molecules. Red indicates positive correlation and blue indicates negative correlation. The first 3 strongest associations are shown by dot plots. 


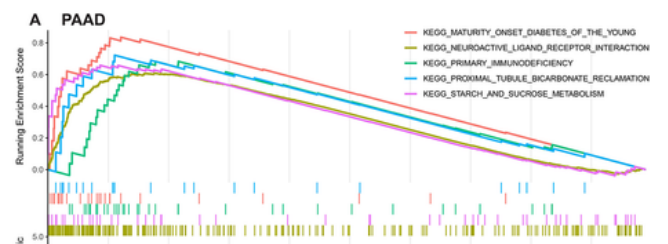

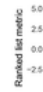

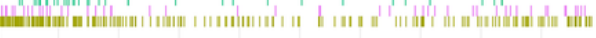

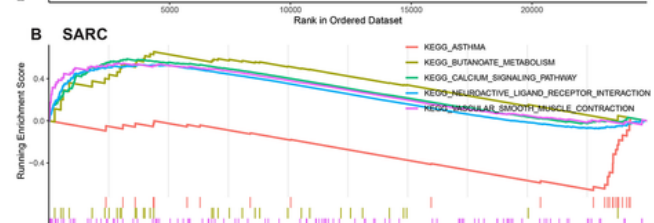

量

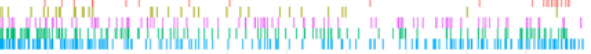
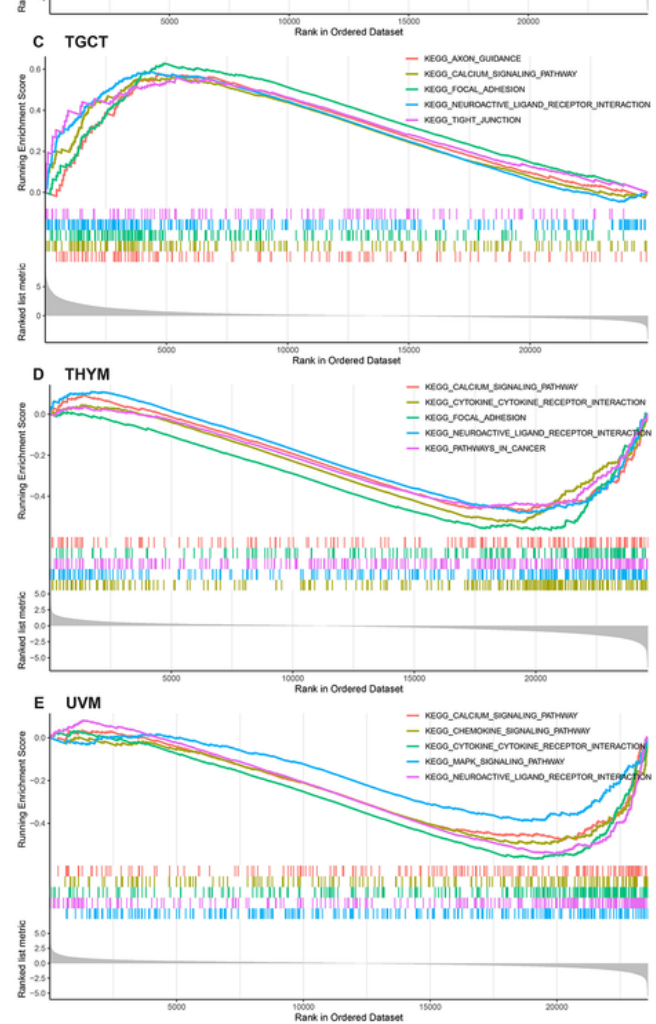

Figure 9

The results of GSEA. KEGG pathway analysis of EPHX2 in multiple cancers.

The different color curves represent different pathways regulated in different tumors. The peak on the upward curve indicates positive adjustment, and the peak on the downward curve indicates negative adjustment. 


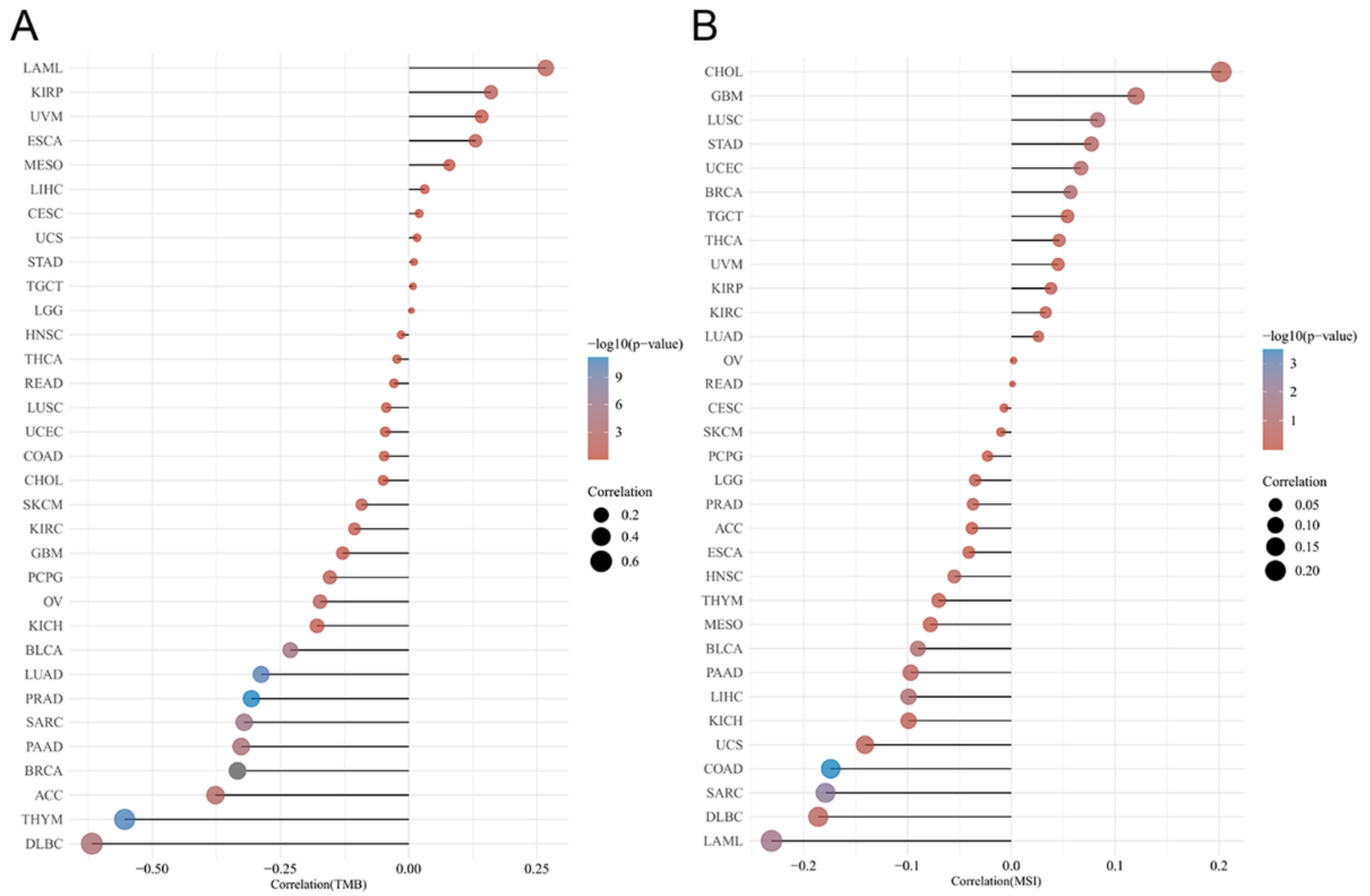

\section{Figure 10}

The correlation between the EPHX2 expression and immunotherapy biomarkers. A Spearman correlation analysis of TMB and EPHX2 gene expression. The horizontal axis in the figure represents the correlation coefficient between genes and TMB, the ordinate is different tumors, the size of the dots in the figure represents the size of the correlation coefficient, and the different colors represent the significance of the $P$ value. The bluer the color, the smaller the $P$ value. B Spearman correlation analysis of MSI and EPHX2 gene expression. The horizontal axis in the figure represents the correlation coefficient between genes and MSI, the ordinate is different tumors, the size of the dots in the figure represents the size of the correlation coefficient, and the different colors represent the significance of the $P$ value. The bluer the color, the smaller the $P$ value. 

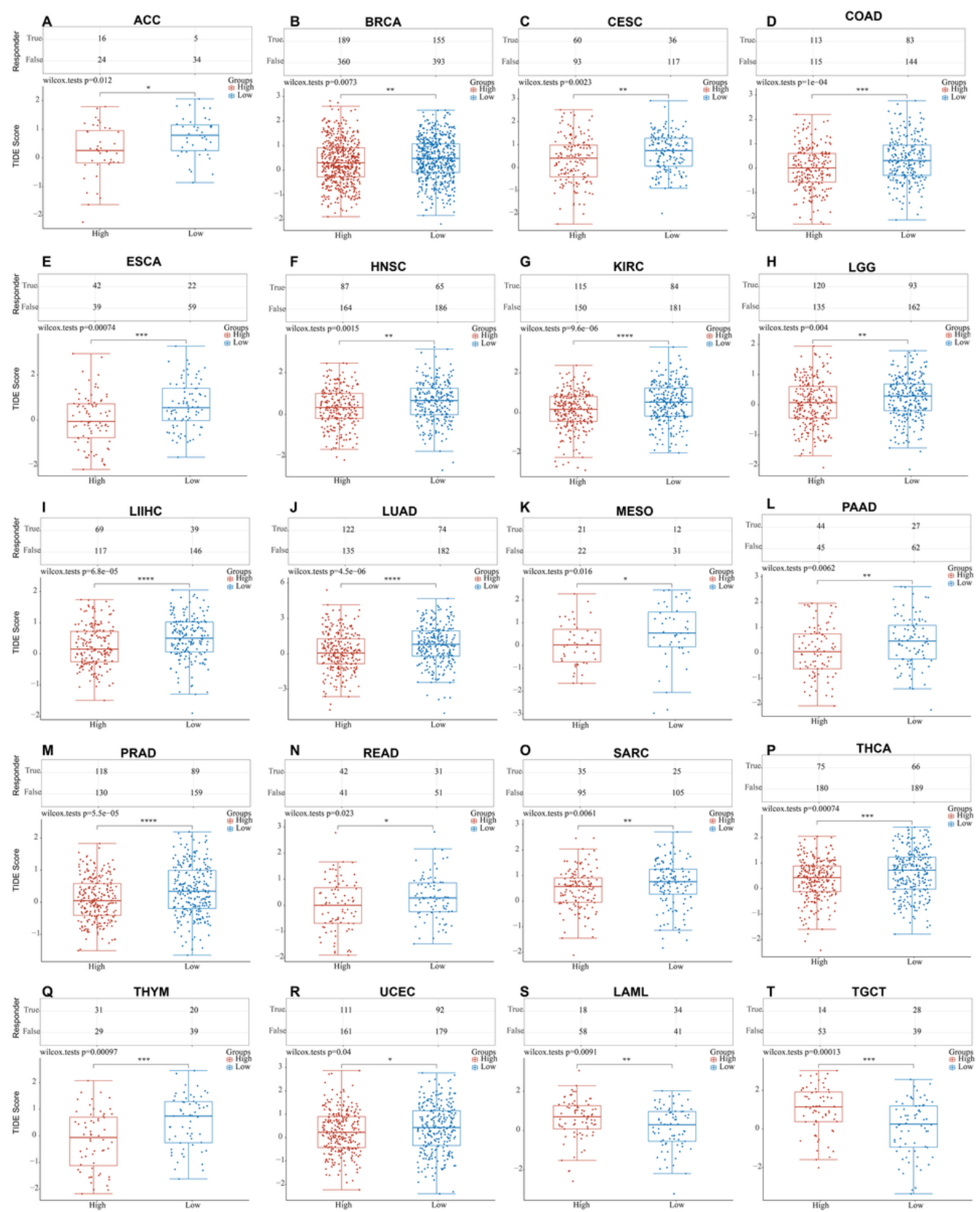

\section{Figure 11}

The TIDE score of EPHX2 expression in TCGA tumors. A-T Above: statistical table of immune response of samples in different groups in the prediction results, Below: the distribution of immune response scores in different groups in the prediction results. Red represents high expression group, blue represents low expression group $\left({ }^{\star} P<0.05, * \star P<0.01, * \star *<0.001\right)$.

\section{Supplementary Files}

This is a list of supplementary files associated with this preprint. Click to download.

- SupplementaryFigure1.pdf

- SupplementaryFigure2.pdf

- SupplementaryFigure3.pdf

- SupplementaryFigureLegends.docx

- SupplementaryTable1.docx 